2014

\title{
Optimization Of Confocal Laser Induced Fluorescence In A Plasma
}

\author{
R. VanDervort \\ D. Elliott \\ D. McCarren \\ J.McKee \\ M. Soderholm \\ See next page for additional authors
}

Follow this and additional works at: https://researchrepository.wvu.edu/faculty_publications

\section{Digital Commons Citation}

VanDervort, R.; Elliott, D.; McCarren, D.; McKee, J.; Soderholm, M.; Sears, S.; and Scime, E., "Optimization Of Confocal Laser Induced Fluorescence In A Plasma" (2014). Faculty Scholarship. 627.

https://researchrepository.wvu.edu/faculty_publications/627 
Authors

R. VanDervort, D. Elliott, D. McCarren, J. McKee, M. Soderholm, S. Sears, and E. Scime 


\title{
Optimization of Confocal Laser Induced Fluorescence in a Plasma ${ }^{\text {a) }}$
}

\author{
R. VanDervort, D. Elliott, J. McKee, D. McCarren, M. Soderholm, S. Sears, and E. \\ Scime ${ }^{\text {b) }}$
}

Department of Physics, West Virginia University, Morgantown, West Virginia 26506-6315, USA

(Presented XX June 2014; received XXXXX; accepted XXXXX; published online XXXXX)

\begin{abstract}
Laser Induced Fluorescence (LIF) provides measurements of flow speed, temperature, and density of ions or neutrals in a plasma. Traditionally, a LIF measurement requires two ports on a plasma device; one for laser injection and one for emission collection. Proper alignment of LIF optics is time consuming and sensitive to mechanical vibration. We describe a confocal configuration for LIF that requires a single port and requires no alignment. The measurement location is scanned radially by physically moving the entire optical structure. Confocal LIF measurements are compared to traditional LIF measurements over the same radial range.
\end{abstract}

\section{INTRODUCTION}

First applied to the diagnosis of plasmas over three decades ago by Stern and Johnson, ${ }^{1}$ laser induced fluorescence (LIF) has evolved into a powerful tool capable of providing non-intrusive, high resolution, spatially resolved, measurements of particle velocity distributions in many types of plasma discharges and for a wide range of experimental conditions., 2,3,4,5 Typically, LIF measurements are performed at a single location in a plasma. However, some groups have developed planar LIF measurement techniques in which a laser beam is spread into a sheet and used to illuminate a cross section of the plasma. ${ }^{6,7,8}$ The induced fluorescence is then imaged with a camera, and a two-dimensional image of LIF intensity and flow along the direction of the laser beam is obtained.

Both measurement techniques require one port in the plasma vacuum vessel for injection of light and one, or more, for light collection. In a low temperature laboratory plasma experiment, enough ports are usually available that LIF measurements are easily accomplished. However, in magnetically confined fusion plasma experiments, plasma access is at a premium and coupled with the complex geometries of such systems, obtaining ports with overlapping lines-of-sight is problematic. Our research group also often travels to other research laboratories where we perform LIF measurements using our portable LIF apparatus. For such experimental campaigns, optical access and the labor-intensive process required for optical alignment of the injection and collection optics, even for experimental facilities with good optical access, adds considerable time to the LIF measurement process.

Confocal fluorescence spectroscopy and imaging is a common technique in biological laboratories and only requires a single optical path. In a confocal microscope (see Fig. 1), a specimen that has been tagged with a fluorescing molecule is illuminated by a light source that passes through the optics of the imaging system and is prevented from coupling into the detection or imaging system by wavelength selective transmissive or reflective optics. ${ }^{9}$ Spatial localization of the LIF measurement in a confocal microscope is accomplished with a high-magnification, short focal length, low $f$-number lens. The fluorescent emission is proportional, when not saturated, to the intensity of the illuminating laser and therefore the measurement location is localized to the focal point of the imaging lens. In a plasma experiment, it is not possible to use a short focal length lens for emission collection. The meas-

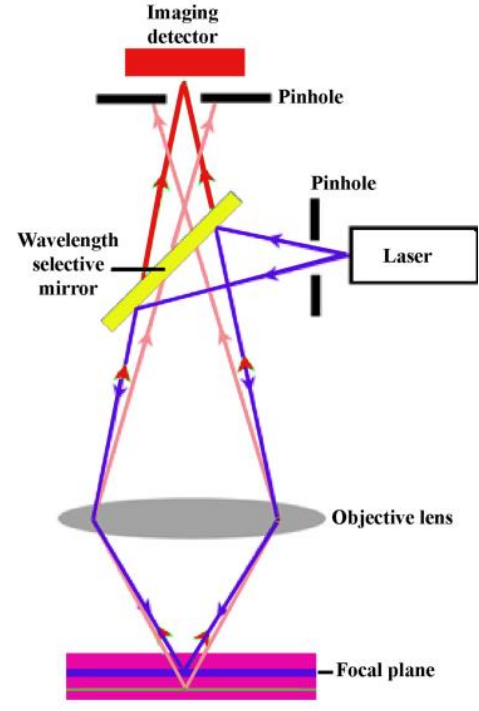

FIG. 1. Schematic of a typical confocal microscope.

urement location is often many centimeters, or even meters, away inside a vacuum chamber filled with high temperature plasma more than capable of destroying any in-situ optics. Spatial localization of LIF measurements is somewhat easier in two-photon LIF experiments because the fluorescent emission is proportional to the square of the laser intensity. We have demonstrated in previous experiments that large $f$-number imaging optics are able to provide two-photon LIF measurements with sub-cm spatial resolution at focal distances of tens of centimeters inside a plasma. ${ }^{10}$

In this work, we describe an optical fiber coupled, confocal LIF system that employs spatial filtering to tightly constrain the spatial extent of the measurement location in a plasma. The entire apparatus is portable, intrinsically self-aligned, and provides sub-cm resolution LIF measurements.

\section{EXPERIMENTAL APPARATUS}

The LIF measurements were performed in argon plasmas created in the Hot hELicon eXperiment (HELIX). ${ }^{11}$ Typical electron temperatures and densities in the steady state-plasma are $T_{\mathrm{e}} \approx 4 \mathrm{eV}$

a) Contributed paper published as part of the Proceedings of the 20th Topical Conference on High-Temperature Plasma Diagnostics, Atlanta, Georgia, June 2014. b) Author to whom correspondence should be addressed. Electronic mail: escime@wvu.edu. 
and $n \sim 1 \times 10^{13} \mathrm{~cm}^{-3}$. All measurements reported here were performed in the helicon source. A complete descriptions of the experimental apparatus may be found in Ref. [12].

A LIF measurement consists of sweeping the frequency of a narrow bandwidth laser through the absorption line of a collection of ions or atoms that have a thermally broadened velocity distribution function. The ions or atoms absorb a photon and are pumped into an excited state when the laser appears at the appropriate frequency in their respective rest frames. Measurement of the intensity of the photon emission from the excited state as a function of laser frequency characterizes a typical LIF measurement. For these argon ion velocity distribution function (ivdf) measurements, the ring dye laser-based LIF laser system (see Ref. [13]) is tuned to $611.662 \mathrm{~nm}$ (vacuum wavelength) to pump the $\mathrm{Ar}$ II $3 \mathrm{~d}^{2} \mathrm{G}_{9 / 2}$ metastable state to the $4 \mathrm{p}^{2} \mathrm{~F}_{7 / 2}$ state, which then decays to the $4 \mathrm{~s}^{2} \mathrm{D}_{5 / 2}$ state by emitting $460.96 \mathrm{~nm}$ photons. ${ }^{14}$ As the laser frequency is swept over as much as $60 \mathrm{GHz}$, the fluorescent emission from the excited state is recorded with a filtered ( $1 \mathrm{~nm}$ bandwidth) narrowband, high-gain, Hamamatsu photomultiplier tube (PMT).

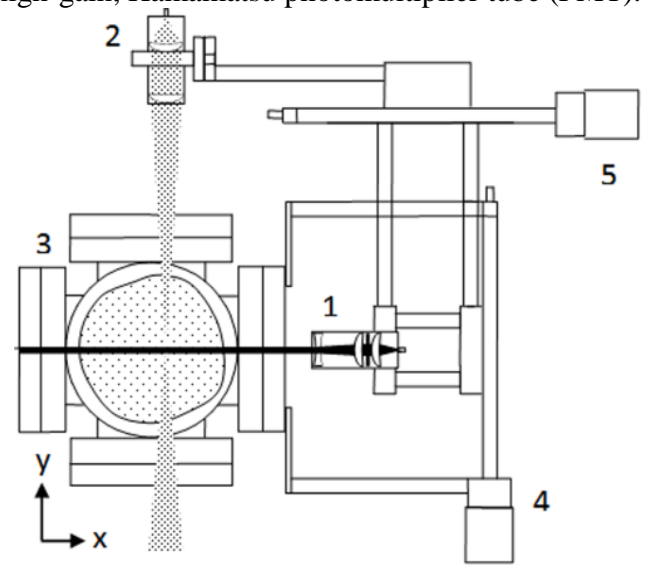

FIG. 2. The crossed lines-of-sight, two-dimensional scanning LIF apparatus: 1 . beam reducing and polarizing injection optics, 2. optimized collection optics, 3. plasma source chamber, 4. $Y$ axis scanning stage, 5. $X$ axis scanning stage.

For validation of the confocal technique, we simultaneously performed spatially resolved LIF measurements of metastable ion density and ion temperature using a conventional, two-dimensional (2D) apparatus. The collection and injection optics for the 2D measurements are mounted on two independent, but coupled, stepping motor stages. The collection optics (Fig. 2) consist of a multimode fiber cable coupled to a $2.54 \mathrm{~cm}$ o.d. collimating lens with a matching numerical aperture $(\mathrm{NA}=0.22)$ to maximize light collection from the collection lens. The $2.54 \mathrm{~cm}$ o.d. collection lens is mounted in front of the collimating lens and the waist of the collection spot is $0.075 \mathrm{~cm}$ at the location of the injected laser beam. The injection optics consist of another $2.54 \mathrm{~cm}$ o.d. collimating lens, followed by a linear polarizer with its polarization axis aligned along the magnetic field, and a beam reducing Galilean telescope. The collimated injection beam has a diameter of $0.5 \pm 0.05 \mathrm{~cm}$ across the entire plasma column. It is this scale that sets the radial resolution of the measurements. When the LIF interrogation volume $\left(\sim 3.9 \times 10^{-3} \mathrm{~cm}^{3}\right)$ is scanned in the vertical direction, both the injection and collection optics move as a single unit; providing a fixed sample volume as the $x$ component of ion velocity, $V_{i x}\left(x_{o}, y\right)$, is measured as a function of $y$ position.

The linear polarizer in the injection optics reduces the injected laser intensity by a factor of 2 . However, by only pumping the $\pi$ transitions $(\Delta m=0)$ in the $\mathrm{Ar}$ II $3 \mathrm{~d}^{2} \mathrm{G}_{9 / 2}$ to $4 \mathrm{p}^{2} \mathrm{~F}_{7 / 2}$ transition sequence, the much larger Zeeman splitting of the $\sigma$ transitions $(\Delta m$ \pm 1 ) lines are avoided and the ivdf can be fit with a single thermally broadened Gaussian function. The internal Zeeman splitting of the $\pi$ lines, Stark broadening, the natural line width of the absorption line, and the laser line width are ignorable. Ivdf width measurements as a function of laser power indicate that power broadening due to saturation of the absorption line ${ }^{15}$ is ignorable.

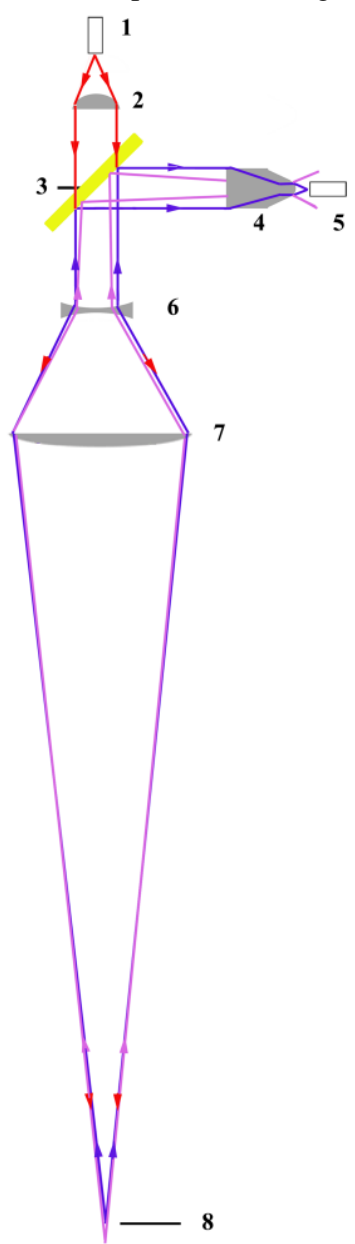

FIG. 3. The confocal LIF apparatus. 1. single mode injection fiber, 2. collimating lens, 3 . low pass dichroic mirror, 4 . x40 microscope objective, 5 . single mode collection fiber, 6 . divergent lens, 7 . objective lens, 8 . focal plane. The spacing of the divergent and objective lenses is not to scale.

The confocal optical design is shown in Figure 3. Spatial resolution of the measurements is obtained by using the largest objective lens ( $5 \mathrm{~cm}$ diameter) practical given the optical access, expanding the injection beam to fill the objective lens, and using a 5 $\mu \mathrm{m}$ core fiber in the collection path as a spatial filter to limit the depth of the field around the focal plane. The factor of 40 magnification microscope objective and the small core of the single mode collection fiber tightly constrain the location of the focal plane. In a typical LIF system, the collection spot is located at the focal point of the objective lens. In our design, the $2.54 \mathrm{~cm}$ divergent lens places the image of the $5 \mu \mathrm{m}$ core of the collection fiber at twice the focal length of the objective lens, $20 \mathrm{~cm}$ away in this case. Not shown in Fig. 3 is a linear polarizer in the injection path to eliminate unwanted Zeeman components as is the 2D system. The dichroic mirror reflects the $460.96 \mathrm{~nm}$ fluorescence while letting pass the $611.6612 \mathrm{~nm}$ pump laser. Fully illuminating the objective lens with the collected light ray paths is critical to maximizing the signal-to-noise of the confocal apparatus.

LIF ion metastable density and ion temperature measurements obtained with the 2D system are shown in Fig. 4. Operating conditions that yielded a sharply peaked metastable density profile were intentionally selected for testing of the confocal apparatus. 


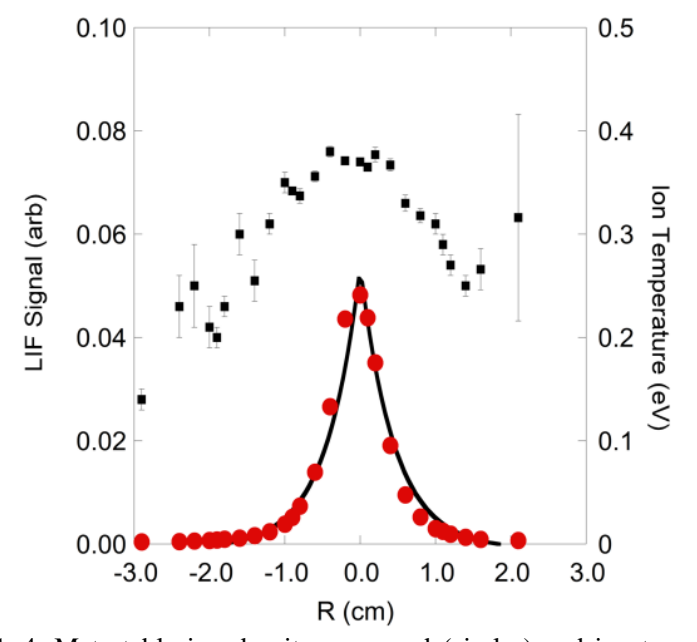

FIG. 4. Metastable ion density measured (circles) and ion temperatures (squares) measured with the 2D apparatus. The solid line is an exponential fit to metastable ion density measurements.

\section{CONFOCAL LIF MEASUREMENTS}

A typical ivdf measurement obtained with the confocal apparatus is shown in Fig. 5a. The signal-to-noise of the measurement is excellent. Radially resolved, confocal measurements of the metastable ion density and the ion temperature, calculated from the width of the Doppler broadened ion absorption line, are shown in Figure 5b.The radial profiles in the confocal measurements are much less steep than those obtained with the 2D apparatus. Whereas in the 2D measurements the ion temperature is roughly constant at $0.38 \mathrm{eV}$ from only -1 to $1 \mathrm{~cm}$, the confocal ion temperature profile is nearly constant at the same $0.38 \mathrm{eV}$ value over the entire measurement range. Thus, it is clear that the confocal apparatus is integrating along the confocal line-of-sight. To determine the integration length scale, the 2D metastable measurements in Fig. 4 were fit with an exponential function and then integrated over a fixed length assuming uniform collection of light along the integration length. Shown in Fig. 5b is the result of such an integration assuming an integration length scale of $3 \mathrm{~cm}$. The confocally measured metastable density profile is well fit by the integrated higher-resolution measurements.

\section{SUMMARY}

These measurements demonstrate the feasibility of performing confocal LIF measurements in a plasma. While the spatial resolution of the measurement is modest, the excellent signal-to-noise suggests that additional gains in spatial resolution are realizable

\section{REFERENCES}

${ }^{1}$ R. A. Stern and J. A. Johnson III, Phys. Rev. Lett. 341548 (1975).

${ }^{2}$ D. Hill, S. Fornaca, and M. Wickham, Rev. Sci. Instrum. 54, 309 (1983).

${ }^{3}$ D. J. Trevor, et al., Appl. Phys. Lett. 57, 1188 (1990).

${ }^{4}$ J. Amorim, et al., J. Phys. D: Appl. Phys., 33, R51 (2000).

${ }^{5}$ A. Keesee, E. Scime, and R. Boivin, Rev. Sci. Instrum. 75, 4091 (2004).

${ }^{6}$ A. D. Bailey, R. Stern, and P. Bellan, Phys. Rev. Lett. 71, 3123 (1993).

${ }^{7}$ F. M. Levinton and F. Trintchouk, Rev. Sci. Instrum. 72, 898 (2001).

${ }^{8}$ N. B. Moore, W. Gekelman, P. Pribyl, Y. Zhang, M. J. Kushner, Phys. Plasmas 20, 083506 (2013)

${ }^{9}$ S. Nie, D.T. Chiu, R.N. Zare, Science 2661018 (1994).

${ }^{10}$ R. M. Magee, M. E. Galante, D. McCarren, E. E. Scime, R. L. Boivin, N. H. Brooks, R. J. Groebner, D. N. Hill, and G. D. Porter, Rev. Sci. Instrum. 83, 10D701 (2012). through additional spatial filtering (at the expense of light collection efficiency). The confocal apparatus is intrinsically aligned, requires only one optical port on a vacuum chamber, and is easily installed on any experimental system in a matter of minutes. The optical fiber coupling of both the injection and collection light enables the user to place sensitive lasers and detectors at considerable distances from the plasma source.

\section{ACKNOWLEDGMENTS}

This work was supported by NSF award PHY-0611571.
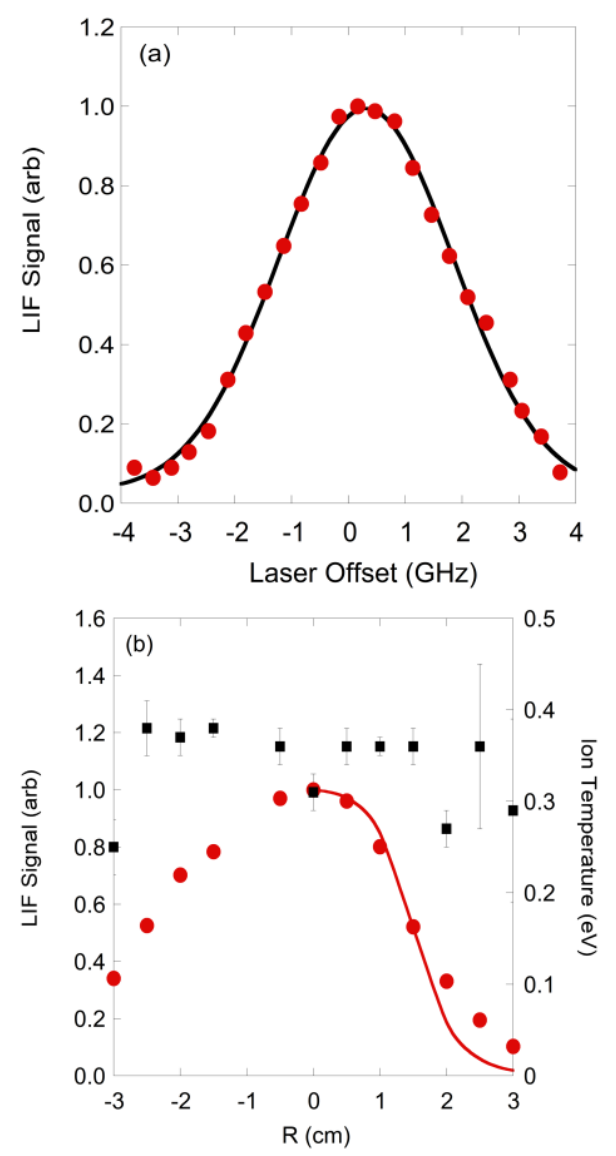

FIG. 5. A) Metastable ion density versus laser frequency shift from the center of the absorption line. The solid line is a Gaussian fit. B) Metastable ion density measured (circles) and ion temperatures (squares) measured with the confocal apparatus. The solid line is an integration of the $2 \mathrm{D}$ data assuming a spatial resolution of the confocal system of $3 \mathrm{~cm}$.

${ }^{11}$ J. L. Kline, E. E. Scime, R. F. Boivin, A. M. Keesee, and X. Sun, Plasma Sources Sci. Technol. 11, 413 (2002).

${ }^{12}$ E. E. Scime, P. A. Keiter, M. M. Balkey, J. L. Kline, X. Sun, A. M. Keesee, R. A. Hardin, I. A. Biloiu, S. Houshmandyar, S. Chakraborty Thakur, J. Carr Jr., M. Galante, D. McCarren, and S. Sears, to appear in J. of Plasma Phys. (2014).

${ }^{13}$ E.E. Scime, P.A. Keiter, M.W. Zintl, M.M. Balkey, J.L. Kline, and M.E. Keopke, Plasma Sources Sci. Technol. 7186 (1998).

${ }^{14}$ C. Biloiu, X. Sun, E. Choueri, C. Compton, F. Doss, J. Heard, E.E. Scime, R. Specktor, and D. Ventura, Plasma Sources Sci. Technol. 14 766 (2005).

${ }^{15}$ M. Goeckner, J. Goree, and T. Sheridan, Rev. Sci. Instrum. 64, 996 (1993). 